\title{
TERAPIA OCUPACIONAL EM DOENÇAS NEUROLÓGICAS E OS SIGNIFICADOS DO SERVIÇO AMBULATORIAL PRESTADO NA PERSPECTIVA DO USUÁRIO
}

\author{
TERAPIA OCUPACIONAL EN ENFERMEDADES NEUROLÓGICAS Y EL \\ SIGNIFICADO DEL SERVICIO AMBULATORIO PROPORCIONADO DESDE LA \\ PERSPECTIVA DEL USUARIO
}
OCCUPATIONAL THERAPY IN NEUROLOGICAL DISEASES AND THE MEANINGS OF AMBULATORY SERVICE PROVIDED FROM THE USER'S PERSPECTIVE

\section{Carolina Albuquerque Alves ${ }^{1}$, Camila Boarini dos Santos ${ }^{2}$, Rita de Cássia Tibério Araújo $^{3}$, Aila Narene Dahwache Criado Rocha ${ }^{4}$}

\begin{abstract}
RESUMO
O presente estudo objetivou identificar como o paciente neurológico, em atendimento no setor de Terapia Ocupacional (T.O), percebe a influência desse serviço em sua saúde global. O estudo foi desenvolvido em um Centro Especializado em Reabilitação II (CER II), de uma cidade de médio porte do interior de São Paulo, que dispõe do serviço de Terapia Ocupacional em Neurologia Ambulatorial Adulto e Idoso. O instrumento utilizado para a coleta de dados foi um roteiro de perguntas semiestruturado, aplicado na forma de entrevista. Foram abordados tópicos para a caracterização dos participantes e para o registro de suas percepções. Os temas destacados abrangeram: como o usuário se sente com o tratamento; quais sentimentos se destacam nesse processo; mudanças percebidas no decorrer das intervenções; e grau de satisfação com o serviço. A análise de dados foi realizada de forma qualitativa por meio dos dados provindos das entrevistas. Houve a prevalência do sexo masculino ( $n=10)$, na faixa etária acima de 18 anos e menor de 60 ( $n=8$ ), com maior incidência de Acidente Vascular Encefálico ( $n=6$ ). Nas questões abertas foi possível identificar a prevalência de sentimentos positivos, a percepção do usuário sobre a contribuição positiva da T.O em relação ao ganho de independência, qualidade de vida e que todos os participantes recomendariam o serviço ofertado pela T.O. O
\end{abstract}

1 Terapeuta Ocupacional. Residente pelo Programa Multiprofissional em Saúde Mental da Universidade Estadual Paulista "Júlio de Mesquita Filho", Faculdade de Medicina de Botucatu - São Paulo, Brasil. ResearcherID: AAN-1450-2020. ORCID: 0000-0002-2181-1633. E-mail: carolinaalves.to@outlook.com. (55 014)99811-1467.

2 Terapeuta Ocupacional. Mestranda junto ao Programa de Pós-Graduação em Educação. Pesquisadora do Laboratório de Acessibilidade, Tecnologia Assistiva e Inclusão (latai) da Faculdade de Filosofia e Ciências da Universidade Estadual Paulista "Júlio de Mesquita Filho" - UNESP - Campus de Marília - São Paulo, Brasil. il. ResearcherID: M-5341-20. ORCID: 0000-0001-5594-0305. E-mail: camilaboarini@hotmail.com. (55 016) 99145-0844.

3 Terapeuta Ocupacional. Professora Doutora do Curso de Terapia Ocupacional - Departamento de Fisioterapia e Terapia Ocupacional da Faculdade de Filosofia e Ciências da Universidade Estadual Paulista "Júlio de Mesquita Filho" -UNESP- campus de Marília -São Paulo, Brasil. ResearcherID: M-5392-2018. ORCID: 0000-0002-1463-1175. E-mail: ritac@marilia.unesp.br. (55 014) 981179772

4 Terapeuta Ocupacional. Professora Doutora do Curso de Terapia Ocupacional - Departamento de Fisioterapia e Terapia Ocupacional. Coordenadora do Laboratório de Acessibilidade, Tecnologia Assistiva "Júlio de Mesquita Filho" - UNESP - campus de Marília - São Paulo, Brasil. ResearcherID: M-5368-2018. ORCID: 0000-0001-6186-875X. E-mail: aila@marilia.unesp.br. (55 014) 99848-6330. Inclusão (latai) da Faculdade de Filosofia e Ciências da Universidade Estadual Paulista "Júlio de Mesquita 
estudo possibilitou um feedback sobre a intervenção prestada e demonstrou que o significado da intervenção contempla a complexidade da base holistica do tratamento.

\title{
PALAVRAS-CHAVE
}

Terapia Ocupacional. Neurologia. Perspectiva do paciente. Reabilitação.

\section{RESUMEN}

El objetivo de la presente investigación es evaluar como el paciente neuropata, en cuidados por la Terapia Ocupacional (T.O), percibe la importancia del servicio en su salud general. La investigación fue desarrollada en el Centro Especialista en Rehabilitación II (CER II), ubicado en una ciudad del departamento de São Paulo, el centro cuenta con un ambulatorio de terapia ocupacional neurológica para adultos y ancianos. Para recolectar los informes fue puesta en práctica una encuesta con un cuestionario semiestructurado, fueron abordados temas con el fin de caracterizar y registrar sus visiones con las siguientes cuestiones principales: ¿Cómo se siente el paciente con el tratamiento recebido?, ¿Cuáles son los sentimientos destacados en este proceso?, los cambios percibidos por el paciente hacia las citas y el grado de satisfacción con el servicio. El análisis de datos fue hecho cualitativamente con los datos recibidos de la encuesta. Hubo un predominio del sexo masculino $(n=10)$, en el grupo de edad mayor de 18 años y menor de $60(n=8)$, con mayor incidencia de accidente vascular encefálico $(n=6)$. En las preguntas abiertas fue posible identificar el predominio de sentimientos positivos, la percepción del paciente sobre la contribución positiva de la T.O en relación con la ganancia de independencia, calidad de vida y que todos los participantes recomendarían el servicio ofrecido por la T.O. La investigación proporcionó un feedback positivo sobre la intervención y demostró que el significado de la intervención, aborda la complejidad de la base holística del tratamiento.

\section{PALABRAS CLAVE}

Terapia ocupacional. Neurología. Perspectiva del Paciente. Rehabilitación

\begin{abstract}
The following study aimed to identify how the neurological patient, who is receiving care in the occupational herapy (O.T.) sector, realizes on this service's influence in their global health. The study was developed in a Specialized Centerfor Rehabilitation II (CER II), Located in a mediumsized town in the interior of São Paulo, which has an Occupational Therapy in Adult and Elderly Outpatient Neurology Service. The instrument used for data collection was a semi-structured questionnaire, applied as an interview. Topics for characterizing the participants and recording their perceptions were addressed. The highlighted topics covered: how the userfeels about the treatment; what feelings stand out in this process; changes perceived in the course of activities; and degree of satisfaction with the service. Data analysis was performed qualitatively based on the information from the interviews. There was a prevalence of males $(n=10)$ aged over 18 and under $60(n=8)$, with a higher incidence of stroke $(n=6)$. In the open questions it was possible to identify positive feelings prevalence, the user's perception of the positive contribution of O.T. regarding gaining independence and quality of life and that all participants would recommend the service offered by O.T. The study provided feedback on the intervention given and showed that the intervention meaning fulfills the complexity of holistic basis of treatment.
\end{abstract}

\section{KEYWORDS}

Occupational Therapy. Neurology. Patient Preference. Rehabilitation.

Recibido:06/03/2019

Aceptado: 06/10/2020 


\section{INTRODUÇÃO}

As doenças neurológicas são caracterizadas pelo acometimento do sistema nervoso central ou periférico, que abrangem perturbações do cérebro, da medula espinhal, dos nervos periféricos e da junção neuromuscular (Huse \& Lucas, 2002).

Segundo o Ministério da Saúde (2008) as doenças neurológicas têm alcançando impacto epidemiológico relevante, considerando sua prevalência e sequelas existentes, cuja sua interferência social é intensa, além de uma elevada taxa de mortalidade.

Atualmente observou-se um aumento na ocorrência de sequelas consequentes as disfunções neurológicas em adultos, em virtude ao envelhecimento populacional, avanços da medicina e à maior exposição aos fatores de risco como tabagismo, consumo nocivo de álcool, inatividade física e má alimentação (Faria, 2007).

As sequelas neurológicas consistem em uma série de déficits e perdas como a perda do controle voluntário dos movimentos musculares, déficits sensoriais, incontinência, dificuldades na comunicação e na fala, que acometem a saúde física, emocional e social (Oliveira, Lucena \& Echer, 2014).

Segundo Othero \& Ayres (2014) com o acometimento de uma doença grave existem rupturas, modificações nos papéis, dor e a redução na participação social em diferentes contextos como familiar e social. No entanto, por mais limitante que seja o cotidiano desse sujeito o mesmo não pode permanecer sem o sentido da vida e resgatar as atividades consideradas significativas é fundamental.

Em decorrência das sequelas o usuário é encaminhado para um serviço de reabilitação que visa à restauração das capacidades e habilidades pessoais da forma mais completa, em que se busca resgatar ao máximo suas habilidades acometidas pela doença (Silva Junior, 1958). Com uma abordagem mais ampliada, a reabilitação contempla a promoção da saúde, a prevenção e a redução das incapacidades, a fim de que as potencialidades são retomadas e, assim, se viabiliza uma vida com mais qualidade, auto-estima e independência (Leite \& Faro, 2005).
Essa visão ampliada vem ao encontro da prática do Terapeuta Ocupacional na área da neurologia, uma vez que o cuidado na área profissional consiste em ações para minimizar as incapacidades, através do estímulo e desenvolvimento adequado das habilidades necessárias para um bom desempenho ocupacional nas atividades básicas e significativas para aquele usuário (Stoffel \& Nickel, 2013).

O terapeuta ocupacional atua na perspectiva do processo saúde-doença, com a atenção dirigida para as atividades que de alguma forma foram interrompidas pelo processo de adoecimento e questionando sobre qual as probabilidades dessas atividades retornarem à vida dessa pessoa (Othero \& Ayres, 2014).

A repercussão que as sequelas geram tanto na independência quanto na qualidade de vida do sujeito é o alvo de restauração no processo de reabilitação conduzido pelo terapeuta ocupacional. De acordo com a Organização Mundial de Saúde (1948) a saúde do sujeito não só será afetada na ocorrência de doenças, mas também pela incapacidade e dificuldades vivenciadas em realizar as atividades significativas após o acontecimento da doença, pois a saúde se configura pelo completo bem-estar físico, mental e social de uma pessoa, o que interfere na qualidade de vida.

De acordo com a Organização Mundial de Saúde (1994) qualidade de vida refere-se à percepção do sujeito quanto a sua inserção no contexto da cultura e sistemas de valores aos quais ele pertence e em relação aos seus objetivos, expectativas, padrões e preocupações de saúde. Tal premissa vem ao encontro dos fundamentos da Terapia Ocupacional em que as funções ocupacionais são importantes para o sujeito de forma integral (Dahdah \& Carvalho, 2014).

Segundo a Classificação Internacional de Funcionalidade, Incapacidade e Saúde (2004) a incapacidade é a dificuldade para o desempenho em atividades, e resulta da relação entre capacidade funcional da pessoa e demandas ambientais.

O conceito de saúde mais amplo contempla o usuário de acordo com seu contexto social, emocional, físico e espiritual apontando para a importância da reabilitação apoiar-se em uma abordagem holística, levando em conta a importância da autonomia e autoestima para a recuperação da rotina de vida e participação social 
(Pereira, Teixeira \& Santos, 2012). E, em se tratando de alteração funcional neurológica permanente, com graus variados de recuperação das funções motoras e percepto-cognitivas, há necessidade de cuidados em saúde que contemplem a motivação do usuário para o seu engajamento na vida, empoderamento frente ao tratamento, observando-se as contra-indicações de atividades, evitando fadiga e piora na qualidade de vida, além de estar de acordo com a realidade frente a intervenção (Vaz, Jubilini \& Queiroz, 2017).

A atuação do terapeuta ocupacional junto aos usuários do serviço, dentre os quais o usuário com sequelas neurológicas valoriza a sua participação na condução do seu tratamento e de sua própria história, mesmo com as limitações presentes, por meio do resgate, exploração e atividades que validem as potências de vida desse sujeito e consequentemente proporcionar a ele autonomia e independência (Othero \& Ayres, 2014).

A área de Terapia Ocupacional realiza ações no modelo de reabilitação com foco nas prioridades do sujeito, integrando-o nas decisões do processo terapêutico, de maneira que as abordagens associam procedimentos biomecânicos aos percepto-cognitivos, em um contexto de valorização da sua saúde mental (Golisz \& Toglia, 2002; Jackson, Gray \& Zemke, 2005).

Nessa perspectiva, a Terapia Ocupacional pode abranger uma variedade de significados e conhecer as dimensões da representação do serviço vem ao encontro dos fundamentos da profissão, possibilitando avaliar o impacto das ações oferecidas no processo de tratamento do paciente.

\section{Objetivo Geral}

Este estudo tem por objetivo identificar como o usuário do serviço, em atendimento no setor de Terapia Ocupacional em Neurologia Ambulatorial Adulto e Idoso, percebe a influência desse serviço em sua saúde global.

\section{Objetivo EsPecífico}

1) Identificar se os usuários possuem a percepção das condutas e dos enfoques da Terapia Ocupacional que são contemplados na sistematização do atendimento prestado.
2) Avaliar o grau de satisfação do usuário em relação ao serviço prestado.

\section{Metodologia}

Este estudo é de caráter qualitativo. Foi submetido à avaliação pelo Comitê de Ética em Pesquisa (CEP) da Faculdade de Filosofia e Ciências da UNESP - Campus de Marília/SP, respeitando as prerrogativas da resolução 510/16 do CONEP que versa sobre ética em pesquisa com seres humanos, possuindo parecer favorável sob o CAEE: 17937319.4.0000.5406 sob o protocolo 3.640.500.

O estudo foi desenvolvido em uma cidade do interior de São Paulo, em uma Unidade Auxiliar de Ensino da Universidade Estadual Paulista (UNESP), denominada Centro Especializado em Reabilitação II (CER II), que dispõe do serviço de Terapia Ocupacional no setor de Reabilitação Física.

Após aprovação pelo CEP, foi realizado contato com os participantes no dia determinado para atendimento, e lhes foi feito o convite para participar da pesquisa, mediante esclarecimentos sobre os objetivos, procedimentos de coleta de dados, tempo de duração, resguardo da privacidade do participante e utilização dos dados para fins científicos, sendo após os esclarecimentos, convidados a assinar o termo de consentimento livre e esclarecido, confirmando anuência.

Participaram da pesquisa 13 usuários atendidos no setor de Terapia Ocupacional em Neurologia Ambulatorial Adulto e Idoso, sendo que a amostra contemplou todos os pacientes atendidos neste setor, exceto aqueles que apresentaram dificuldade de compreensão para responder o questionário de forma independente, dentre os quais, pacientes diagnosticados com Doença de Alzheimer, que devido a evolução do quadro clínico não puderam participar do presente trabalho.

As características da amostra em dados percentuais quanto à idade, sexo, diagnóstico e tempo de tratamento encontram-se apresentadas na Tabela 1. 
TABELA 1 CARACTERIZAÇÃO GERAL DA AMOSTRA

\begin{tabular}{l|l}
\hline CARACTERIZAÇÃO GERAL DA AMOSTRA & FREQUÊNCIA \\
\hline IDADE (média em anos) & 51 \\
SEXO & 3 \\
Feminino & 10 \\
Masculino & \\
DIAGNÓSTICO & 6 \\
Acidente Vascular Encefálico (AVE) & 2 \\
Esclerose múltipla & 1 \\
Parkinson & 2 \\
Traumatismo Crânio Encefálico (TCE) & 1 \\
Plexo Braquial & 1 \\
Anterolistese 1 do corpo vertebral C4 & \\
ENCAMINHAMENTO PARA T. O & 2 \\
Fisioterapeuta & 3 \\
Conhecido & 6 \\
Médico & 2 \\
Terapeuta Ocupacional & 91 \\
TEMPO DE DOENÇA (média em meses) & 25 \\
TEMPO DE TRATAMENTO NA T.O (média em meses) &
\end{tabular}

Fonte: Elaboração própria

\section{FIGURA 1 ESCALA VISUAL ANALÓGICA.}

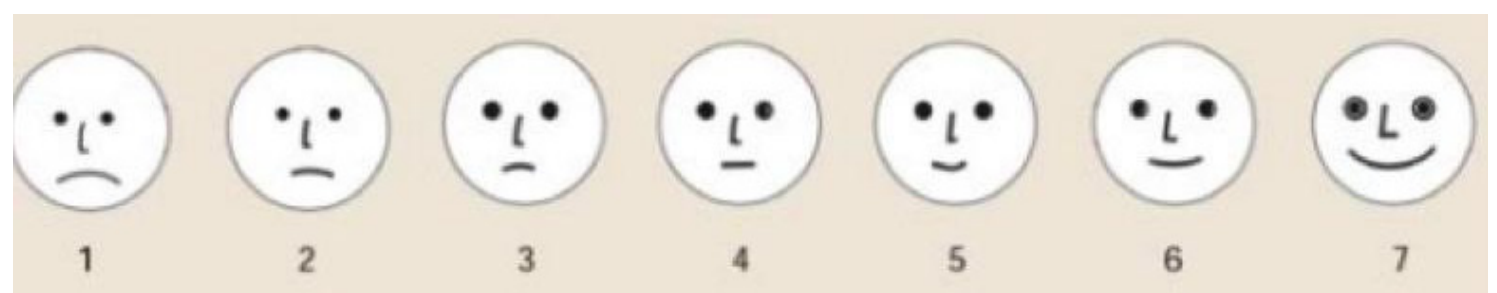

Fonte: Indicadores na Gestão. Bachmann \& Associados

O instrumento utilizado para a coleta de dados foi um roteiro de perguntas semiestruturado elaborado pelas autoras e aplicado na forma de entrevista. O roteiro foi intitulado "Avaliação sobre o serviço de Terapia Ocupacional na perspectiva do usuário" e abordou tópicos para a caracterização dos participantes e para o registro de suas percepções quanto aos sentimentos em relação ao tratamento realizado tendo como destaque perguntas sobre como o usuário se sente com o tratamento, quais sentimentos se destacam nesse processo e mudanças percebidas no decorrer das intervenções. 
Utilizou-se também da escala visual analógica (Figura 1) para indicar o nível de satisfação com o serviço prestado pela Terapia Ocupacional.

O questionário foi avaliado por dois juízes da área que realizaram considerações sobre o instrumento utilizado. Após realizada as adequações, elaborou-se o roteiro final. $\mathrm{Na}$ qual, as respostas eram transcritas durante a entrevista.

A análise de dados foi realizada de forma qualitativa por meio dos dados provindos das entrevistas, utilizando a análise de conteúdo proposta por Bardin (2016). Optou-se pela análise de conteúdo do tipo categorial, pois permite dividir as respostas em categorias de acordo com o objeto de referência citado na fala do usuário entrevistado (Bardin, 2016).

\section{Resultados e Discussão}

Os dados apreciados quanto à caracterização dos participantes demonstram que a média de idade dos participantes foi de 51 anos, com variação de idade máxima 81 anos e mínima de 23 anos, e prevalência do sexo masculino, sendo 10 dos 13 entrevistados. Os diagnósticos apresentados pelos participantes incluíram o de AVE em 6 dos entrevistados, em seguida de Esclerose Múltipla com 2, Traumatismo Crânio Encefálico com 2, Plexo Braquial 1 e Anterolistese 1 do corpo vertebral C4. Os encaminhamentos ao setor em sua maioria ocorreram por médicos com 6 usuários do serviço, seguido de conhecidos com 3 usuários, fisioterapeutas 2 e terapeutas ocupacionais com 2. A média do tempo da ocorrência da patologia foi 91 meses, tendo como mínimo 2 meses e máximo 22 anos. Em relação ao tempo de tratamento na Terapia Ocupacional a média foi 25 meses, sendo que o mínimo foi de um mês em tratamento e o máximo de 6 anos (Quadro 1).

QUADRO 1. CARACTERIZAÇÃO DOS PARTICIPANTES.

\begin{tabular}{|c|c|c|c|c|c|c|}
\hline PARTICIPANTES & SEXO & IDADE & DIAGNÓSTICO & $\begin{array}{l}\text { TEMP. } \\
\text { DOENÇA }\end{array}$ & $\begin{array}{l}\text { TEMPO DE } \\
\text { TRATAMENTO NA T.O. }\end{array}$ & $\begin{array}{l}\text { ENCAMINHADO } \\
\text { POR: }\end{array}$ \\
\hline P1 & M & 73 anos & AVE & $\begin{array}{l}1 \text { ano e } 8 \\
\text { meses }\end{array}$ & 1 ano e 4 meses & Conhecido \\
\hline P2 & M & 52 anos & $\begin{array}{l}\text { Esclerose } \\
\text { Múltipla }\end{array}$ & 22 anos & 6 anos & Médico \\
\hline P3 & M & 81 anos & AVE & 5 anos & 3 meses & Conhecido \\
\hline $\mathbf{P 4}$ & M & 61 anos & AVE & 21 anos & 2 anos & Conhecido \\
\hline P5 & M & 76 anos & Parkinson & 18 anos & 2 anos & Médico \\
\hline P6 & $\mathrm{F}$ & 91 anos & AVE & 2 meses & 1 mês & $\begin{array}{l}\text { Terapeuta } \\
\text { Ocupacional }\end{array}$ \\
\hline P7 & M & 50 anos & TCE & 2 anos & 1 ano e 6 meses & Fisioterapeuta \\
\hline P8 & $\mathrm{F}$ & 23 anos & AVE & 5 anos & 4 anos & Médico \\
\hline P9 & M & 24 anos & Plexo braquial & 3 anos & 1 ano & Médico \\
\hline P10 & M & 32 anos & TCE & 11 anos & 4 anos & Médico \\
\hline P11 & M & 53 anos & $\begin{array}{l}\text { Esclerose } \\
\text { Múltipla }\end{array}$ & 10 anos & 1 ano & Médico \\
\hline P12 & M & 50 anos & AVE & 5 anos & 3 anos & $\begin{array}{l}\text { Terapeuta } \\
\text { Ocupacional }\end{array}$ \\
\hline P13 & $\mathrm{F}$ & 55 anos & $\begin{array}{l}\text { Anterolistese } \\
1 \text { do corpo verte- } \\
\text { bral C4 }\end{array}$ & 5 anos & 1 ano & Fisioterapeuta \\
\hline
\end{tabular}

Fonte: Elaboração própria. 
No presente estudo houve a prevalência do sexo masculino $(n=10)$ o que difere do estudo de Viana et al., (2013) em que predominou a participação do sexo feminino. Viana et al (2013) atribuem a baixa procura aos serviços de saúde por homens, devido às dificuldades em aderir práticas voltadas ao autocuidado, ou a associação de que a procura pelos serviços está ligada a sentimentos de fraqueza, medos e inseguranças. Levando em conta os dados do presente estudo, pode-se atribuir a maior frequência do sexo masculino à própria incidência do AVE nessa população.

Em relação ao tempo de doença e o tempo de tratamento na Terapia Ocupacional a maior parte dos participantes do presente estudo $(n=10)$ demoraram de um ano ou mais em relação ao tempo de doença para chegarem ao setor de Terapia Ocupacional e assim iniciar o tratamento. Apesar das diferenças entre o porte da cidade do presente estudo e a cidade do artigo de Spedo, Pinto \& Tanaka (2010) ao questionar possíveis justificativas sobre as dificuldades para acessar os serviços especializados na cidade de São Paulo obteve como resultado em sua pesquisa três categorias, sendo que duas destas categorias vão de encontro a situação dos participantes, englobam a temática de distância dos serviços especializados e a demora para conseguir atendimento. Mesmo que a cidade em questão seja de menor porte as dificuldades para se chegar ao atendimento estão alinhadas as categorias elencadas por Spedo, Pinto \& Tanaka (2010) pois os usuários precisam percorrer longas distancias visto que muito dos participantes são de cidades próximas, demandam de transporte público para chegar ate o CER e a longa fila de espera para chegarem ao atendimento, o que dificulta o acesso ao serviço.

A faixa etária prevalente de usuários maiores de 18 anos e menores de 60 anos na amostra, está em concordância com o estudo de Toldrá \& Sá (2005), o qual teve como objetivo analisar a qualidade de um serviço Ambulatório de Terapia Ocupacional, a partir da visão do usuário, tendo afirmado que essa parcela representada se diferencia pela produtividade e por serem ativos economicamente.

Segundo o Ministério da Saúde (2008), as doenças neurológicas interferem socialmente na produtividade do usuário acometido, o que corrobora com esta pesquisa, visto que dos 13 participantes, 8 estão entre a faixa etária de 18 anos e 60 anos, os mesmos não realizam nenhuma atividade laboral. Dessa forma, se faz necessário utilizar-se de estratégias que abordem não só os procedimentos de reabilitação funcional dos usuários, mas também de estratégias que contemplem essa fase produtiva do sujeito por meio de aspectos laborais e socioculturais (Toldrá \& Sá, 2005).

Em relação às doenças identificadas, destacou-se a notável incidência do Acidente Vascular Encefálico (AVE) na amostra de participantes avaliada, o que corrobora com o dado apresentado pelo Ministério da Saúde (2013) em que o AVE representa, no Brasil, a primeira causa de morte e incapacidade. Sendo que o número de jovens e adultos acometidos aumenta devido à exposição cada vez maior aos fatores de risco como a inatividade física, uso de contraceptivo oral, uso excessivo de bebidas alcoólicas, obesidade e o tabagismo (Costa et al., 2008).

A via mais evidente de encaminhamento ao setor de Terapia Ocupacional foi por meio de profissionais da área da saúde $(n=10)$, com a prevalência dos encaminhamentos realizados por médicos, sendo estes responsáveis por 6 dos encaminhamentos presentes na amostra da pesquisa. Por ser tratar de um Centro Especializado em Reabilitação (CER) conveniado ao Sistema Único de Saúde é um serviço regulado, territorial, caracterizado como lugar de referência no cuidado e proteção para usuários, familiares e acompanhantes nos processos de reabilitação de acordo com Portaria 793/12 (Freitas, 2016). Esses dados apontam para a importância da inserção do serviço de TO na rede SUS, dando visibilidade às suas ações que, centralizadas nas necessidades do paciente de forma individualizada, estão em harmonia com o pressuposto de saúde do SUS.

Em relação à como se sentem com o tratamento todos os participantes atribuíram sentimentos positivos, com as respectivas justificativas (Quadro 2). 


\section{QUADRO 2. SENTIMENTOS COM O TRATAMENTO}

\begin{tabular}{|l|l|}
\hline P1 & $\begin{array}{l}\text { Outros: ótimo, me sinto bem, sou bem } \\
\text { tratado. }\end{array}$ \\
\hline P2 & $\begin{array}{l}\text { Bem - converso, me divirto, vejo muita } \\
\text { gente. }\end{array}$ \\
\hline P3 & $\begin{array}{l}\text { Bem - sinto melhora, só de chegar já está } \\
\text { fazendo alguma coisa. }\end{array}$ \\
\hline P4 & Bem \\
\hline P5 & Bem e Motivado \\
\hline P6 & Bem - é uma família. \\
\hline P7 & Bem \\
\hline P8 & Bem \\
\hline P9 & $\begin{array}{l}\text { Motivado - o estímulo dado pela T.O. me } \\
\text { ajudou a realizar as atividades que não ima- } \\
\text { ginava mais como amarrar o sapato, escre- } \\
\text { ver. O tempo ajuda a se adaptar. }\end{array}$ \\
\hline P10 & $\begin{array}{l}\text { Motivado - com o tratamento consegui vol- } \\
\text { tar a ser ativo. }\end{array}$ \\
\hline P11 & $\begin{array}{l}\text { Motivado - Uma hora maravilhosa que eu } \\
\text { passo aqui. Durante o dia não converso tan- } \\
\text { to quanto aqui. }\end{array}$ \\
\hline P12 & $\begin{array}{l}\text { Motivado - gosto de vir aqui, conhecer gen- } \\
\text { te nova, mudança. }\end{array}$ \\
\hline P13 & Motivado - quero melhorar, ficar boa. \\
\hline
\end{tabular}

Fonte: Elaboração própria.

Os sentimentos relatados a como se sentem com o tratamento foram bem-estar geral ( $\mathrm{P}_{2}, \mathrm{P}_{3}, \mathrm{P}_{4}, \mathrm{P}_{5}, \mathrm{P}_{6}, \mathrm{P}_{7} \mathrm{e}$ $\mathrm{P} 8)$, motivado ( $\mathrm{P}_{5}, \mathrm{P}_{9}, \mathrm{P}_{10}, \mathrm{P}_{11}, \mathrm{P}_{12}$ e $\left.\mathrm{P}_{13}\right)$ e outros caso os sentimentos presentes não fossem os ideais como ótimo ( $\left.\mathrm{P}_{1}\right)$. Nas justificativas dos sentimentos atribuídos ao tratamento destacaram: a participação social $\left(\mathrm{P}_{2}, \mathrm{P} 6\right.$ e $\left.\mathrm{P}_{11}\right)$, retornar a ser ativo $\left(\mathrm{P}_{10}\right.$ e $\left.\mathrm{P}_{13}\right)$, acolhimento $\left(\mathrm{P}_{1}\right)$ e a realização de atividades especificas $(\mathrm{Pg})$, sendo que $\mathrm{P}_{4}, \mathrm{P}_{5}, \mathrm{P}_{7}$ e $\mathrm{P} 8$ não justificaram suas escolhas.

Em relação aos sentimentos manifestados, ao se lembrarem do atendimento, os participantes entrevistados selecionaram sentimentos positivos, e alguns participantes escolheram mais de um sentimento. Os sentimentos incluíram a sensação de bem-estar geral $\left(\mathrm{P}_{2}\right.$, $\left.\mathrm{P}_{5}, \mathrm{P} 6, \mathrm{P}_{10}, \mathrm{P}_{11} \mathrm{E} \mathrm{P}_{13}\right)$, alegria ( $\mathrm{P}_{3}, \mathrm{P}_{4}, \mathrm{P}_{7}, \mathrm{P}_{8}, \mathrm{P}_{11}$ e $\left.\mathrm{P}_{13}\right)$, entusiasmo $\left(\mathrm{P}_{5}, \mathrm{P} 8, \mathrm{P}_{9}\right.$ e $\left.\mathrm{P}_{13}\right)$ e gratidão $\left(\mathrm{P}_{1}\right)$. De uma maneira geral, os sentimentos relatados com relação ao tratamento coincidiram com os sentimentos relatados ao lembrarem do atendimento. Pode-se considerar como fator de importância para os sentimentos manifestados o papel do uso terapêutico de si, em que o terapeuta desenvolve e gerencia sua relação terapêutica com o usuário (Taylor \& Van Puymbroeck, 2013). Cole \& McLean (2003) enfatizam que, segundo os usuários, o resultado da intervenção se apoia na relação do usuário com o terapeuta.

Esses resultados evidenciam a importância do acolhimento e da ambiência do serviço ao longo do processo de tratamento, pois é necessário que os usuários desfrutem de um ambiente que lhes seja saudável, confortável, que respeite sua privacidade, que ainda consiga viabilizar um local para o encontro e uma assistência de forma individualizada, reconhecendo suas peculiaridades e necessidades de saúde, de forma a sustentar a relação entre serviço e usuário (Brasil, 2006; Brasil, 2010; Brasil, 2013).

Nessa perspectiva e dentro dos pressupostos da Terapia Ocupacional (Samea, 2008), a fala do participante 11 sintetiza a importância do setting terapêutico, sobre o bem-estar geral do paciente.

\section{P11 - "Uma hora maravilhosa que eu passo aqui. Durante o dia não converso tanto quanto aqui".}

Em relação às mudanças existentes em sua qualidade de vida, após iniciar o tratamento, todos os sujeitos afirmaram melhora na qualidade de vida com as respectivas justificativas (Quadro 3).

\section{QUADRO 3. MUDANÇAS NA QUALIDADE DE VIDA APÓS INICIAR A TERAPIA OCUPACIONAL.}

\begin{tabular}{|l|l|}
\hline P1 & Eu não levantava o braço, hoje já consigo. \\
\hline P2 & $\begin{array}{l}\text { Fico mais contente/alegre, não consigo } \\
\text { explicar esse sentimento, tenho tempo para } \\
\text { cuidar de mim }\end{array}$ \\
\hline P3 & Diminuiu a dor, estou andando melhor. \\
\hline P4 & Melhora na mobilidade. \\
\hline P5 & Relaxo mais e corrijo a postura. \\
\hline P6 & $\begin{array}{l}\text { Quero corresponder ao tratamento e me } \\
\text { esforço. }\end{array}$ \\
\hline P7 & $\begin{array}{l}\text { Comecei ajudar no banho, colocar a roupa e } \\
\text { vir na T.O de andador. }\end{array}$ \\
\hline
\end{tabular}




\begin{tabular}{|l|l|}
\hline P8 & $\begin{array}{l}\text { Com a T.O. consegui voltar a realizar ativida- } \\
\text { des como colocar a calça, abrir o zíper e cor- } \\
\text { tar alimentos que antes eu não conseguia. }\end{array}$ \\
\hline P9 & $\begin{array}{l}\text { A dificuldade em dar nó, escrever foi supera- } \\
\text { da a partir da T.O. }\end{array}$ \\
\hline P10 & $\begin{array}{l}\text { Antes estava acomodado, sem rumo tomar } \\
\text { e com o tratamento consegui retomar a vida } \\
\text { com mais independência, consigo me virar } \\
\text { (sem ficar pedindo). }\end{array}$ \\
\hline P11 & Fiquei mais espontâneo, mais feliz. \\
\hline P12 & $\begin{array}{l}\text { Minha mão não abria e hoje ela está melhor. } \\
\text { Com as adaptações (tábua de carne, cortador } \\
\text { de unha) consigo me virar. }\end{array}$ \\
\hline P13 & $\begin{array}{l}\text { O braço melhorou, quase não sinto dor e } \\
\text { sinto que melhorou bem. }\end{array}$ \\
\hline
\end{tabular}

Fonte: Elaboração própria.

Quando abordados sobre a qualidade de vida, todos os sujeitos relataram qualidade de vida melhorada sob aspectos da mobilidade ( $\left.\mathrm{P}_{1}, \mathrm{P}_{4}, \mathrm{P}_{7}, \mathrm{P}_{10}\right)$, de realização de atividades específicas, sendo atividades de vida diária e atividades instrumentais de vida diária (AVD e AIVD) $\left(\mathrm{P}_{2}, \mathrm{P} 7, \mathrm{P} 8, \mathrm{P} 9\right)$, motivação para a resolução de problemas (P2, P6, P10), adaptação à condição de vida ( $\left.\mathrm{P}_{5}, \mathrm{P}_{11}\right)$, diminuição da dor ( $\left.\mathrm{P}_{3}, \mathrm{P}_{13}\right)$, organização postural $\left(\mathrm{P}_{5}\right)$ e uso de tecnologia assistiva ( $\left.\mathrm{P}_{12}\right)$.

Esses resultados demonstram o efeito favorável do tratamento de forma holística, e representam a conscientização dos participantes quanto aos domínios de intervenção do terapeuta ocupacional (Leite \& Faro, 2005). Quanto ao treino de AVDS e AIVD com pacientes neurológicos foi evidenciado que possibilitaram a aprendizagem para o desempenho das atividades da forma mais independente possível (Cruz, 2010), como evidenciado na fala do P1o.

P1o - "Antes estava acomodado, sem saber que rumo tomar, e com o tratamento consegui retomar a vida com mais independência, consigo me virar (sem ficar pedindo)."

Em relação à opinião dos entrevistados sobre as possíveis influências da Terapia Ocupacional em relação à sua independência, foi confirmada por todos os entrevistados a influência favorável sobre sua independência, as justificativas contemplaram as atividades de vida diária (AVD) e habilidades subjacentes, de natureza motora e emocional, para o desempenho (Quadro 4).

QUADRO 4. INFLUÊNCIA DA T.O EM RELAÇÃO À CONFIRMAÇÃO DE INDEPENDÊNCIA E SUAS JUSTIFICATIVAS.

\begin{tabular}{|l|l|}
\hline P1 & $\begin{array}{l}\text { Me auxiliou a ter mais movimento, a T.O. me } \\
\text { ajuda muito. }\end{array}$ \\
\hline P2 & Eu escolho minhas roupas, sapatos. \\
\hline P3 & Acha que é bom, estou andando melhor. \\
\hline P4 & Na coordenação motora, mobilidade. \\
\hline P5 & Trouxe mais incentivo. \\
\hline
\end{tabular}

Fonte: Elaboração própria.

Quanto às implicações do diagnóstico sobre a independência, também se observa a sinalização das habilidades do desempenho que resultam de estruturas e função corporal (Oliveira, Lucena \& Echer, 2014). E mais importante ainda, verifica-se uma percepção embasada na autonomia, para o enfrentamento das dificuldades do ponto de vista pessoal e controle emocional (Othero \& Ayres, 20I4). Destaque também foi dado às prescrições de equipamentos de tecnologia assistiva que favorecem a funcionalidade, bem como o retorno à participação social (Steultjens et al., 2003), como evidenciado no relato do PI2.

P12 - "Minha mão não abria e hoje ela está melhor. Com as adaptações (tábua de carne, cortador de unha) consigo me virar".

Em relação à contribuição da Terapia Ocupacional de maneira mais pontual, todos afirmaram que sentiram melhoras e justificaram-nas (Quadro 5).

\section{QUADRO 5. CONTRIBUIÇÃO DA TERAPIA OCUPACIONAL}

\begin{tabular}{|l|l|}
\hline P1 & Principalmente pelo movimento, a fala. \\
\hline P2 & Traz um sentimento de bem-estar, livre. \\
\hline P3 & Melhorou as dores, me sinto melhor. \\
\hline P4 & Me sinto bem, principalmente na marcha. \\
\hline P5 & $\begin{array}{l}\text { Fico mais tranquilo e entusiasmado para o } \\
\text { tratamento. }\end{array}$ \\
\hline P6 & $\begin{array}{l}\text { Sempre ajuda, a gente precisa pensar e ficar } \\
\text { pensando. }\end{array}$ \\
\hline P7 & A T.O. me ajudou em tudo. \\
\hline
\end{tabular}




\begin{tabular}{|l|l|}
\hline P8 & $\begin{array}{l}\text { Antes da T.O. eu dependia muito da minha } \\
\text { mãe e irmã, a partir da T.O. me senti mais } \\
\text { independente, comecei a tentar fazer as } \\
\text { coisas. }\end{array}$ \\
\hline P9 & $\begin{array}{l}\text { Estou mais adaptado, consegui retomar as } \\
\text { atividades que acreditava ter perdido. }\end{array}$ \\
\hline P10 & $\begin{array}{l}\text { Estou menos desanimado em relação a como } \\
\text { cheguei e mais motivado a ser ativo e melho- } \\
\text { rar. Estou mais disposto. }\end{array}$ \\
\hline P11 & $\begin{array}{l}\text { O tratamento da T.O ajudou em tudo. Fiquei } \\
\text { mais espontâneo, um cara mais solto. }\end{array}$ \\
\hline P12 & $\begin{array}{l}\text { A T.O. contribuiu para que eu estivesse mais } \\
\text { adaptado e assim me sinto bem e motivado } \\
\text { com o tratamento. }\end{array}$ \\
\hline P13 & $\begin{array}{l}\text { Me sinto mais adaptada, consigo fazer os } \\
\text { serviços domésticos, acho que vir na T.O. é } \\
\text { importante para mim. }\end{array}$ \\
\hline
\end{tabular}

Fonte: Elaboração própria.

Em entrevista, todos os participantes afirmaram que, após iniciar o tratamento com a Terapia Ocupacional, obtiveram uma melhor independência na realização de atividades. Entretanto, somente cinco justificaram a melhora: três participantes $\left(\mathrm{P}_{1}, \mathrm{P}_{3}\right.$ e $\left.\mathrm{P}_{4}\right)$ relataram ganho de independência sob aspectos da mobilidade, o poder de escolha $\left(\mathrm{P}_{2}\right)$ e motivação para a vida $\left(\mathrm{P}_{5}\right)$.

Quando questionados sobre seu grau de satisfação com o serviço prestado pela Terapia Ocupacional, 12 dos participantes atribuíram grau máximo e apenas o entrevistado $\mathrm{P}_{5}$ atribuiu grau parcial de satisfação em relação ao serviço.

Segundo Toldrá \& Sá (2005), a satisfação atribuída pelos usuários ao serviço prestado pode ser associada ao reconhecimento dos aspectos técnicos, como pode ser identificado nas respostas a seguir:

$\mathrm{P}_{3}$ - "Diminuiu a dor, estou andando melhor"

P6 - "Relaxo mais e corrijo a minha postura".

P7 - "Comecei a ajudar no banho, colocar a roupa e vir na T.O de andador".

P13 - "O braço melhorou, quase não sinto dor e sinto que melhorou bem".
Além de que a relação interpessoal é outro elemento que usuário utiliza para justificar seu nível de satisfação com o serviço (Toldrá \& Sá, 2005) o que influencia quando o sujeito é questionado se recomendaria a Terapia Ocupacional a outra pessoa.

Em relação à possível recomendação do serviço de Terapia Ocupacional, todos os usuários relataram que recomendariam o serviço (Quadro 6).

\section{QUADRO 6. RECOMENDAÇÃO DA TERAPIA OCUPACIONAL E JUSTIFICATIVAS.}

\begin{tabular}{|l|l|}
\hline P1 & Todos que puder, me tratam muito bem aqui. \\
\hline P2 & $\begin{array}{l}\text { Porque me sinto bem, porque me sinto reali- } \\
\text { zado aqui. Chego aqui e fico a vontade. }\end{array}$ \\
\hline P3 & $\begin{array}{l}\text { Já recomendei, mas ele não quis vir. Se vier } \\
\text { para cá, melhoraria. }\end{array}$ \\
\hline P4 & "Bom para melhorar" \\
\hline P5 & Participante preferiu não responder. \\
\hline P6 & Aconselharia sim, pois foi bom para mim. \\
\hline P7 & $\begin{array}{l}\text { Porque a T.O é legal, ela ajuda em tudo, me } \\
\text { sinto melhor. }\end{array}$ \\
\hline P8 & $\begin{array}{l}\text { Porque é bom, ajuda bastante, senti melhora } \\
\text { e bem-estar. }\end{array}$ \\
\hline P9 & $\begin{array}{l}\text { Ajuda psicologicamente, fisicamen- } \\
\text { te e estimula a tentar aquilo que estava } \\
\text { impossibilitado. }\end{array}$ \\
\hline P10 & $\begin{array}{l}\text { Dialogar com a terapeuta estimula a usar a } \\
\text { cabeça para pensar, agir pela atividade. }\end{array}$ \\
\hline P11 & É muito bom, é maravilhoso. \\
\hline P12 & Porque é bom e me ajudou bastante. \\
\hline P13 & $\begin{array}{l}\text { Porque é bom para a gente, ajuda é impor- } \\
\text { tante para quem precisa. }\end{array}$ \\
\hline
\end{tabular}

Fonte: Elaboração própria.

No presente estudo todos os usuários afirmaram que recomendariam a Terapia Ocupacional sob aspectos como sentimento de contribuição positiva para si ( $\mathrm{P}_{3}$, $\mathrm{P}_{4}, \mathrm{P} 6, \mathrm{P}_{7}, \mathrm{P} 8, \mathrm{P}_{11}, \mathrm{P}_{12}$ e $\left.\mathrm{P}_{13}\right)$, acolhimento (P1), escuta qualificada ( $\mathrm{P}_{10}$ ) e $\mathrm{P}_{5}$ não justificou sua resposta.

Evidencia-se que o reconhecimento técnico, as relações interpessoais, a escuta qualificada, gentileza e a segurança transmitida ao longo de todo o tratamento são importantes para que o usuário se sinta satisfeito com o serviço prestado pela Terapia Ocupacional (Alcântara, 
Amado \& Silva, 2017), como exemplificado por algumas falas dos entrevistados:

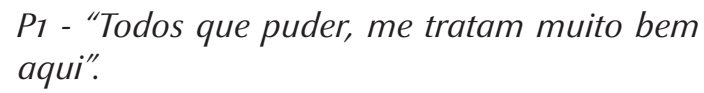

P8 - "Porque ébom, ajuda bastante, senti melhora e bem-estar".

P9 - "Ajuda psicologicamente, fisicamente e estimula a tentar aquilo que estava impossibilitado".

A comunicação interpessoal é um dos principais meios para divulgação do serviço prestado, sendo que, conforme o grau de satisfação aumenta, maior a probabilidade dessa comunicação informal. Quanto mais satisfeito o usuário estiver, maior a chance de que o mesmo possa seguir com seu tratamento e ainda divulgar o serviço prestado (Alcântara, Amado \& Silva, 2017; Santos, Bicalho \& Borges, 2012).

A fala do participante 3, exemplifica a crença do usuário no serviço recebido.

$P_{3}$ - "Já recomendei, mas ele não quis vir. Se vier para cá, melhoraria.".

A partir da avaliação da satisfação do serviço ofertado na perspectiva do usuário, proporciona-se um importante indicador para estimar a qualidade do serviço, sendo, na verdade, uma das principais vias de se repensar e buscar melhorias ao que é ofertado (Paiva, Mendes, Brandão \& Campos, 2015).

\section{CONCLUSÃO}

Conclui-se que é importante valorizar e propiciar momentos de participação ativa do paciente, já que a partir desses estímulos é possível contribuir para o desenvolvimento de autonomia, almejada pela Terapia Ocupacional e preconizada pelo Sistema Único de Saúde (Toldrá \& Sá, 2005).

O presente estudo possibilitou identificar a percepção favorável das ações da Terapia Ocupacional com usuário com sequelas neurológicas, pois, estes participantes percebem a influência do serviço para a melhora de sua saúde global. Dentre as contribuições para a melhora da qualidade de vida do usuário ao longo do processo de reabilitação, foram destacadas a autonomia, a independência, a motivação, a satisfação pessoal.

Sugere-se a partir deste estudo, a necessidade de novas pesquisas, que proporcionem espaço ao usuário, para que o mesmo possua voz ativa em relação ao processo de tratamento, sentimentos existentes e suas, expectativas. Sugere-se ainda que pesquisas posteriores englobem um número maior de participantes, pois são encontrados poucos estudos que visam descrever a percepção, participação do usuário frente ao serviço prestado e que busquem avaliar o grau de satisfação em relação ao atendimento de Terapia Ocupacional.

Neste estudo não foi possível oferecer esclarecimentos sobre o participante $\mathrm{P}_{4}$, que atribuiu uma nota parcial para a sua avaliação em relação à satisfação com o serviço, sem justificativa. Contudo, a pesquisa aponta para a necessidade de melhor entender as necessidades deste paciente e assim viabilizar espaços que contribuam para a participação do usuário.

\section{REFERÊNCIAS BIBLIOGRÁFICAS}

Alcântara, E. B., Amado, C. F. \& Silva, A. C. D. (2017). O CUIDADO EM TERAPIA OCUPACIONAL NA PERSPECTIVA DE USUÁRIOS DE UMA UNIDADE DE SAÚDE DA FAMÍLIA. João Pessoa. Trabalho de Conclusão de Curso (Bacharel em Terapia Ocupacional) Universidade Federal do Paraíba.

Bardin, L. (Ed.70). (2016). Análise Conteúdo. São Paulo.

Ministério da Saúde Brasil. (2. ed.). (2010). Secretaria de Atenção à Saúde. Núcleo Técnico da Política Nacional de Humanização. Acolhimento nas práticas de produção de saúde. Brasília. 5. Reimp. Editora do Ministério da Saúde. (Série B. Textos Básicos de Saúde, 44).

MINISTÉRIO DA SAÚDE Brasil. (2008). Neurologia/Neurocirugia. Recuperado de http://www.saude.gov.br/atencao-especializadae-hospitalar/especialidades/neurologia-neurocirurgia.

Ministério da Saúde Brasil. (2013). Secretaria de Atenção à Saúde. Departamento de Ações Programáticas Estratégicas. Diretrizes de atenção à reabilitação da pessoa com acidente vascular cerebral. Brasília. Recuperado de http://bvsms.saude.gov.br/bvs/publicacoes/ diretrizes atencao reabilitacao acidente vascular cerebral.pdf. Ministério da Saúde Brasil. (3. Ed.). (2006). Política Nacional de Humanização (PNH): HumanizaSUS - Documento-Base. Brasília.

Ministério da Saúde Brasil. (2013). Política Nacional de Humanização - PNH. Brasília.

Cole, B. \& Mclean, V. (2003). Therapeutic relationships rede-fined. Occupational Therapy in Mental Health, 19, 33-56. Recuperado de http://dx.doi.org/10.1300/J004v19n02_03 
COSTA, F. et al. (2008). Nível de conhecimento da população adulta sobre acidente vascular cerebral (AVC) em Pelotas - RS. Curitiba. Jornal Brasileiro Neurocirurgia, 19 (1), 31-37.

Cruz, D.M.C. (2010). Preditores de independência funcional nas atividades de vida diária pós-acidente vascular encefálico. São Carlos. Cadernos de Terapia Ocupacional da UFSCar, 18(3), 275-86.

Dahdah, D.F \& Carvalho, A.M.P. (2014). Papéis ocupacionais, benefícios, ônus e modos de enfrentamento de problemas: Um estudo descritivo sobre cuidadoras de idosos dependentes no contexto da família. São Carlos. Cad. Ter. Ocup. UFSCar, 22(3), 463-472. Recuperado de http://dx.doi.org/10.4322/cto.2014.067.

Faria, I. (2007). Neurologia adulto: disfunções neurológicas. In: CAVALCANTI, A.; GALVÃO, C. Terapia Ocupacional:fundamentação e prática. Rio de Janeiro: Guanabara Koogan. 187- 204.

Leite, V. B. E \& Faro, A.C.M. (2005). O Cuidar do enfermeiro especialista em reabilitação físico-motora. Rev Esc Enferm USP, 39(1), 92-96.

Freitas, A. E. T. D. et al. (2016). DIRETRIZES PARA A ORGANIZAÇÃO DAS AÇÕES DEREABILITAÇÃO NA REDEDECUIDADOS À PESSOA COM DEFICIÊNCIA. São Paulo. Organização: Área Técnica de Saúde da Pessoa com Deficiência. Secretaria Municipal de Saúde.

Golisz, K. M. \& Toglia, J. P. (2002). Avaliação da Percepção e Cognição. In: SPACKMAN. Terapia Ocupacional. Rio de Janeiro: Editora Guanabara, 238-261.

Huse, D. M \& Lucas A. R. (2002). Transtornos comportamentais que afetam a ingestão de alimentos: anorexia nervosa, bulimia nervosa e outras condições psiquiátricas. In: Shils, M.E.; Olson, J. A.; Shike, M. \& Ross, A. C. Tratado de Nutrição Moderna na Saúde e na doença. (9. ed.). São Paulo: Manole. 2106p.

Jackson, J., Gray, J. M. \& Zemke, R. (2005). Otimização de Habilidades e Capacidades: Amplitude de Movimentos, Força e Resistência. In Trombly, C.A., Radomsli, M.V. Terapia Ocupacional para Disfunções Físicas. São Paulo: Livraria Santos Editora. Cap. 20.

Oliveira, M. C., Lucena, A. F. \& Echer, I. C. (2014). Sequelas Neurológicas: Elaboração de um Manual de orientação para cuidado em saúde. Português/Inglês. Rev enferm UFPE on line, Recife, 8(6), 597-603.

Organização Mundial da Saúde. (1995). Avaliação da Qualidade de Vida da Organização Mundial da Saúde (WHOQOL): documento de posicionamento da Organização Mundial da Saúde. Ciências sociais e medicina. 41(10), 403-409.

Organização Mundial da Saúde. (2004). Classificação Internacional de Funcionalidade, Incapacidade e Saúde. Direcção-Geral da Saúde. Lisboa. Recuperado de http://biblioteca.cofen.gov.br/wp-.

Othero, M. B \& Ayres J. R. C. M. (2014). Resgate biográfico como estratégia de assistência. Rev Ter Ocup Univ São Paulo, 25(1), 8087. Recuperado de http://dx.doi.org/10.11606/issn.2238-6149. v25ilp80-7
Paiva M. B. P, Mendes W; Brandão A. L \& Campos C. E. A. (2015). Uma Contribuição para a Avaliação da Atenção Primária à Saúde pela Perspectiva do Usuário. Physis - Revista de Saúde Coletiva, Rio de Janeiro, 25(3), 925-950. Recuperado de https://doi.org/10.1590/ S0103-73312015000300013

Pereira, E. F., Teixeira, C. S. \& Santos, A. (2012). Qualidade de vida: abordagens, conceitos e avaliação. Rev. bras. Educ. Fís. Esporte, São Paulo, 26(2), 241-50.

Samea, M. (2008). O dispositivo grupal como intervenção. Revista de Terapia Ocupacional da USP, São Paulo, 19(2), 85-90. Recuperado de https://doi.org/10.11606/issn.2238-6149.v19i2p85-90

Santos, J. B, Bicalho, K. A \& Borges, M. B. S. (2012). Características e nível de satisfação dos pacientes atendidos na clínica-escola de fisioterapia da Universidade Católica de Brasília. EFDeportes. com, Revista Digital.; 17(171).

Silva J. (1958). Reabilitação de doentes neurológicos. Arq. NeuroPsiquiatr., São Paulo, 16(1), 41-46. Recuperado de http://www. scielo.br/scielo.php?script=sci_arttext\&pid=S0004-282X195 8000100005\&lng=en\&nrm=iso. http://dx.doi.org/10.1590/ S0004-282X1958000100005.

Spedo' S. M, Pinto' N. R. S. \& Tanaka, O. Y. (2010). O difícil acesso a serviços de média complexidade do SUS: o caso da cidade de São Paulo, Brasil. Physis: Revista de Saúde Coletiva. Rio de Janeiro, 20(3), 953-972.

STEULTJENS, E. M. J. et al. (2003). Occupational Therapy for stroke patients: a systematic review. Stroke, Dallas, 34, 676-687. PMid:12624291. Recuperado de http://dx.doiorg/10.1161/01.STR.0000057576.77308.30

Stoffel, D.P. \& Nickel, R. (2013). A utilização da atividade como ferramenta no processo de intervenção do terapeuta ocupacional em reabilitação neurológica. São Carlos. Cad. Ter. Ocup. UFSCar, 21(3), 617-622. Recuperado de http://dx.doi.org/10.4322/cto.2013.064.

Suda, E.Y., Uemura, M.D. \& Velasco, E. (2009). Avaliação da satisfação dos pacientes atendidos em uma clínica-escola de Fisioterapia de Santo André, SP. Fisioterapia e Pesquisa, São Paulo, 16(2), 126-31.

Taylor, R. R., Van Puymbroeck, L. (2013). Therapeutic use of self: Applying the intentional relationship model in group therapy. In O'BRIEN, J. C.; J. W. SOLOMON, J. W. (Eds.), Occupational analysis and group process, 36-52. St. Louis, MO: Elsevier.

Toldrá, R. C. e De Sá, M. J. C. N. (2005). A visão do usuário de um serviço de terapia ocupacional: em busca da qualidade da assistência. Revista de Terapia Ocupacional da USP, São Paulo, 16(2), 90-96.

Vaz, D.V., Jubilini, L.G. \& Queiroz, L.C. (2017). Prática centrada no cliente na reabilitação: definição, instrumentos e desafios. Rev Ter Ocup Univ São Paulo. 28(1), 122-127. Recuperado de http:// dx.doi.org/10.11606/issn.2238-6149.v28ilp122-127. 
Viana, J. R. S.; et. al. (2014). Avaliação da satisfação com a fisioterapia de pacientes atendidos em uma clínica escola de Maringá-PR. Revista UNINGÁ Review, 17(2), 16-21.

WHOQOL Group. (1994). O desenvolvimento do instrumento de avaliação da qualidade de vida da Organização Mundial da Saúde (WHOQOL). In: Orley, J.; Kuyken, W. Avaliação da qualidade de vida: perspectivas internacionais. Heidelberg: Springer, 41-60.

Word Health Organization. (1948). Constituição da Organização Mundial de Saúde. Conferência Internacional da Saúde. New York: WHO. Recuperado de http://www.who.int/governance/eb/ who constitution sp.pdf 
